\title{
Peak Power Demand and Energy Consumption Reduction Strategies for Trains under Moving Block Signalling System
}

\author{
Qing Gu, ${ }^{1}$ Tao Tang, ${ }^{1}$ Fang Cao, ${ }^{1}$ Hamid Reza Karimi, ${ }^{2}$ and Yongduan Song ${ }^{1,3}$ \\ ${ }^{1}$ State Key Laboratory of Rail Traffic Control and Safety, Beijing Jiaotong University, Beijing 100044, China \\ ${ }^{2}$ Department of Engineering, Faculty of Engineering and Science, University of Agder, 4898 Grimstad, Norway \\ ${ }^{3}$ School of Automation, Chongqing University, Chongqing 400044, China \\ Correspondence should be addressed to Yongduan Song; ydsong@bjtu.edu.cn
}

Received 16 July 2013; Accepted 24 September 2013

Academic Editor: Xinjie Zhang

Copyright (c) 2013 Qing Gu et al. This is an open access article distributed under the Creative Commons Attribution License, which permits unrestricted use, distribution, and reproduction in any medium, provided the original work is properly cited.

\begin{abstract}
In the moving block signalling (MBS) system where the tracking target point of the following train is moving forward with its leading train, overload of the substations occurs when a dense queue of trains starts (or restarts) in very close distance interval. This is the peak power demand problem. Several methods have been attempted in the literature to deal with this problem through changing train's operation strategies. However, most existing approaches reduce the service quality. In this paper, two novel approaches"Service Headway Braking" (SHB) and "Extending Stopping Distance Interval" (ESDI) - are proposed according to available and unavailable extra station dwell times, respectively. In these two methods, the restarting times of the trains are staggered and traction periods are reduced, which lead to the reduction of peak power demand and energy consumption. Energy efficient control switching points are seen as the decision parameters. Nonlinear programming method is used to model the process. Simulation results indicate that, compared with ARL, peak power demands are reduced by $40 \%$ and $20 \%$ by applying SHB and ESDI without any arrival time delay, respectively. At the same time, energy consumptions are also reduced by $77 \%$ and $50 \%$ by applying SHB and ESDI, respectively.
\end{abstract}

\section{Introduction}

Moving block signalling (MBS) [1] was proposed a few decades ago to reduce headway among successive trains in a track line. Theoretically, two successive trains are separated by a short distance, which is equivalent to the braking distance of the following train from its current speed, as well as a safety margin. This distance can be changed with the limit for the given operating speed and train characteristics, such as train length and braking rate. In MBS, when a leading train stops for a long time, the following trains will stop at the tail of the leading train. When this leading train restarts, the following trains will start almost simultaneously. It could cause synchronization of the peak demand of trains and increases the total peak power demand significantly, which is highly energy consumed and may lead to overload of the nearby substations. How to reduce peak power demand is called "Peak Demand Reduction" (PDR) problem.

This problem could be solved by improving the infrastructure or the train operation strategies. Energy storage system (ESS) is a very important component in modern railway power supply system. Advanced control and manufacturing technology improve the stability, capacity, and weight of ESS [2-5], which makes it suitable to be equipped in the substations or in trains. It could store the regenerative braking energy and assist the main power source during train's acceleration period. However, it is costly to equip ESS since the whole system needs a large number of them, for example, in urban railway system, nearly each of the stations or trains needs an ESS. At the same time, the cost of maintenance for ESS is also huge, especially when the frequency of acceleration\braking is high (which means the high frequency of charge and discharge and leads to life time reduction). Furthermore, it is inconvenient to be adopted by existing lines since the reconstruction project is costly and complex. Therefore, changing train operation strategies is a more convenient and economical way to reduce peak power demand.

There are two kinds of traditional PDR techniques based on changing operation strategies; one is called starting time 
delay (STD), which introduces a starting time delay to each of the following trains. Under this category, there are two specific techniques called single STD and grade STD. The difference between them is the introduced starting time delay to each of the following trains, which are the same in single STD technique but different in grade STD (showing a deceasing trend). The other one is called acceleration rate limit (ARL), which means the acceleration of the following trains is limited to a certain extent (or different extents). Under this category, there are two specific techniques called single ARL and grade ARL. The difference between them is the limited acceleration rate to each of the following trains, which are the same in ARL technique but different in grade ARL (showing a deceasing trend). In addition to the above techniques, there is also a PDR technique called coordinated PDR. It is a combination of the STD and ARL techniques with feeding the regenerated power of decelerating trains to accelerating trains in the same queue, by coordinating the movement of queued trains.

Takeuchi and his colleagues discussed these techniques in [6-8]. Simulation results show that the graded ARL technique has the best performance in reducing peak power demand among single STD, grade STD, single ARL, grade ARL, and coordinated PDR techniques. In the traditional techniques, time delay is introduced and quality of service is degraded. Ho and Wong [9] use an expert system to help the operators for decision making, and it focuses on the balance between time delay and peak power demand. In [10], by braking and powering the trains simultaneously, Albrecht proposed a way to reduce the peak energy consumption and maximize the regenerative energy. Chen and his colleagues [11] proposed a method to minimize the peak energy consumption by adjusting the train dwell times at each station in MRT systems. Kim et al. [12] developed a mixed integer programming model to minimize the peak power energy demand that occurs when trains are running simultaneously, and the basic principle of the method is the same with STD. All of the methods proposed in [6-12] are implemented after the trains' restarting, by adjusting the timetable or driving strategy.

Although the existing techniques can reduce peak power demand at different degrees, they increase the travel time between the successive stations and decrease the service quality. And the energy consumption is increased since there are more traction periods.

In this paper, in order to reduce peak power demand and energy consumption, we first analyze the reasons of the formation of the peak power demand, and, then, two novel approaches are proposed based on the main reasons with considering the energy efficient driving strategies. One is for available extra station dwell time, named Service Headway Braking (SHB), and the other one is for unavailable extra station dwell time, named Extending Stopping Distance Interval (ESDI). Both of them are real-time adjustment methods and can be implemented before the leading train's restarting. Therefore, there is no need to change timetable. Considering energy saving driving strategy could be seen as a kind of driving mode switching process [13-16]; a nonlinear programming model is contributed and the simulation results show that, compared with the best traditional PDR techniques, both of these two methods could reduce the peak power demand and energy consumption without any arrival time delay increasing. And when the available extra station dwell time is the same as the unavailable extra station dwell time, SHB performs better than ESDI since more peak power and energy consumption could be reduced.

\section{Tracking Dynamics and Peak Power Demand in MBS}

2.1. Tracking Model in MBS. Under MBS, the tracking target point of the following train moves forward continuously as the leading train travels. The instantaneous distance $L_{z}(t)$ of two successive trains is calculated as

$$
L_{z}(t)=S_{\text {leading }}(t)-S_{\text {following }}(t)
$$

where $S_{\text {leading }}(t)$ is the position of the leading train's head and $S_{\text {following }}(t)$ is the position of the following train's head.

The distance between two successive trains must be larger than the safety margin at any moment even if the leading train comes to a sudden halt, so we have

$$
L_{z}(t) \geq L_{\text {safe }}+L_{t}+\frac{V_{\text {following }}(t)^{2}}{2 b},
$$

where $L_{t}$ is the length of the train, $L_{\text {safe }}$ is the length of safety margin, $V_{\text {following }}(t)$ is the instantaneous speed of the following train, and $b$ is deceleration rate.

Based on (1) and (2), the relation between the leading train and following train should satisfy

$$
S_{\text {leading }}(t) \geq L_{\text {safe }}+L_{t}+S_{\text {following }}(t)+\frac{V_{\text {following }}(t)^{2}}{2 b},
$$

which implies that the instantaneous speed and position of the following train should satisfy

$$
\begin{aligned}
& V_{\text {following }}(t) \\
& \quad \leq \sqrt{2 \times b \times\left(S_{\text {leading }}(t)-S_{\text {following }}(t)-L_{\text {safe }}-L_{t}\right)}, \\
& S_{\text {following }}(t) \\
& \quad \leq S_{\text {leading }}(t)-L_{\text {safe }}-L_{t}-\frac{V_{\text {following }}(t)^{2}}{2 b} .
\end{aligned}
$$

2.2. Reasons of the Formation of Peak Power Demand. The reason of the formation of the peak power demand is the restarting of the dense queue, and the reasons for the formation of the dense queue are listed as follows.

(1) Features of moving block signaling system. (Two trains will start simultaneously if the distance interval between them is $L_{\text {safe }}+L_{t}$.)

(2) Extra dwell time in station. 


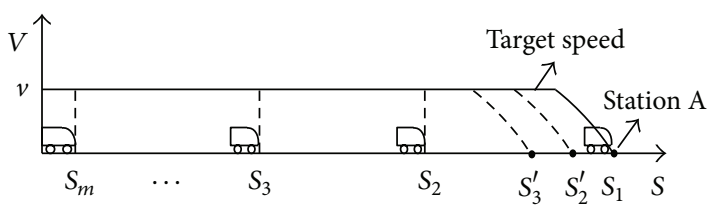

FIGURE 1: Formation of dense queue.

There are two kinds of extra station dwell time: one is available and the other is unavailable. In daily railway operation, there may be some exceptions, such as a passenger may be caught in the door of the train or a short-term surge in passenger flow (i.e., passenger flow increases sharply after a football match). In these circumstances, adjusting the whole timetable is not convenient, because the circumstances only exit in a short period. In this case, the operator will arrange the train to stop a little longer and this extra station dwell time is available. However, if a train is broken in a station and we are not sure how long we need to fix it, in this situation, the extra station dwell time is unavailable.

Based on the analysis above, it is known that peak power demand could be reduced by avoiding the dense queue. In order to achieve this goal, we first analyze the relation between extra station dwell time and the number of delayed trains.

2.3. Station Delay Propagation. Generally speaking, each train has a required dwell time at a station. If a train stops longer than the required dwell time, we call the extra time as delay time. In this section, we focus on the relation between the delay time and the number of delayed trains. In MBS, the delay time may impact the following trains and cause a dense queue. Figure 1 shows the formation of the dense queue.

As it is shown in Figure 1, there are $m$ trains in the track. Train 1 stops at station A. The position of station $\mathrm{A}$ is $S_{1}$. For each train, the dwell time is $T_{\text {dwell }}$. The target speeds of the trains are the same and constant. According to the normal condition, each train arrives at station $A$ and stops for $T_{\text {dwell }}$ and then starts to run.

When train 1 starts, the positions of following trains are $S_{i}, i=2,3, \ldots, m$. The tracking time (the running time of the $i$ th train running from $S_{i}$ to $S_{i-1}$ ) between two successive trains is $\Delta t_{\text {tracking; }}$; it is also the scheduled departure time interval. However, the following trains may become a dense queue if train A does not run immediately after $T_{\text {dwell }}$. Defining $T_{\text {delay }}$ is the delay time of the leading train after $T_{\text {dwell }}$ and $n$ is the number of the delayed trains caused by $T_{\text {delay }}$. Based on (5), train $i$ which is delayed should stop at point $S_{i}^{\prime}$; in other words, $S_{i}^{\prime}$ is the stop position of train $i$ in the dense queue:

$$
S_{i}^{\prime}=S_{i-1}-L_{\text {safe }}-L_{t}
$$

Let $\Delta t_{i}$ be the running time in which train $i$ arrived at $S_{i}^{\prime}$. Based on (2), we have $S_{1}-S_{2}^{\prime}=L_{t}+L_{\text {safe }}, S_{1}-S_{3}^{\prime}=2\left(L_{t}+L_{\text {safe }}\right)$, $S_{1}-S_{4}^{\prime}=3\left(L_{t}+L_{\text {safe }}\right), \ldots, S_{1}-S_{n}^{\prime}=(n-1)\left(L_{t}+L_{\text {safe }}\right)$, and

$$
\begin{aligned}
& \Delta t_{2}=\Delta t_{\text {tracking }}-T_{\text {dwell }}-\left(\left(L_{t}+L_{\text {safe }}\right) / v\right), \Delta t_{3}=2 \times \Delta t_{\text {tracking }}- \\
& T_{\text {dwell }}-2 \times\left(\left(L_{t}+L_{\text {safe }}\right) / v\right), \ldots, \Delta t_{n}=(n-1) \times \Delta t_{\text {tracking }}- \\
& T_{\text {dwell }}-(n-1) \times\left(\left(L_{t}+L_{\text {safe }}\right) / v\right) \text {. } \\
& \quad \text { Letting } T_{\text {delay }} \geq \Delta t_{n} \text {, we have } \\
& \quad T_{\text {delay }} \\
& \quad \geq(n-1) \Delta t_{\text {tracking }}-T_{\text {dwell }}-(n-1) \times \frac{L_{t}+L_{\text {safe }}}{v}
\end{aligned}
$$

then the number of delayed trains should satisfy

$$
n \leq \frac{T_{\text {delay }}+T_{\text {dwell }}}{\Delta t_{\text {tracking }}-\left(\left(L_{t}+L_{\text {safe }}\right) / v\right)}+1 .
$$

2.4. Peak Power Demand Calculation. The power demand of the $i$ th train $P_{i}$ is calculated by

$$
P_{i}=F_{i}(v) \times v_{i}
$$

where $F_{i}(v)$ is the traction force of the $i$ th train and $v_{i}$ is the speed of the $i$ th train after restarting.

The peak power demand of the $i$ th train $P_{i-\text { peak }}$ is calculated by

$$
P_{i-\text { peak }}=\max \left\{P_{i}\right\},
$$

where $F_{i}(t)$ is the traction force of the $i$ th train and $v_{i}(t)$ is the highest speed of the $i$ th train after restarting.

The total peak power demand of all delayed following trains $P_{\text {total }}(t)$ is calculated by

$$
P_{\text {total }}=\sum_{i=1}^{n} P_{i-\text { peak }},
$$

where $n$ is the number of delayed following trains.

2.5. Energy Efficient Control Switching Points. Because there are more traction periods, traditional PRD techniques cause energy consumption increasing. Energy conservation is the research interest in many fields $[17,18]$, especially in rail transport [13-16]. Therefore, it would be better to reduce peak power demand and energy consumption at the same time. Train operation is a switching process; the operation modes are switching among traction, braking, and coasting (with no traction and braking force). There are lots of researches on switching system [19-21]. For optimal control of switching system, we could apply the methodologies proposed in [22, 23]. However, the computation complexity will be a big problem when we adopt the technologies above. In this paper, we first apply Pontryagin maximum principle to find the discrete optimal control modes and then treat the control switching speed as the decision parameters to obtain a reference trajectory.

For electric traction systems, the motion equations of train have the following forms:

$$
\begin{gathered}
\frac{d v(x)}{d x}=\frac{u_{f} f(v)-u_{b} b(v)-r(v)}{v(x)}, \\
\frac{d t(x)}{d x}=\frac{1}{v(x)},
\end{gathered}
$$


where $x$ is the position of the train, $v(x)$ is the speed of the train at position $x, t(x)$ is the time at which the train is located at the position $x, u_{f}$ is the relative traction force, $f(v)$ is the specific maximum traction force per mass unit, $u_{b}$ is the relative braking force, $b(v)$ is the specific maximum braking force per mass unit, and $r(v)$ is the specific basic resistance.

Note that the basic resistance $r(v)$ is usually given by Davis equation:

$$
r(v)=a_{1} v^{2}+a_{2} v+a_{3}
$$

where $a_{1}, a_{2}$, and $a_{3}$ are regression coefficients obtained by fitting test data to the Davis equation. $a_{3}$ accounts for air resistance. $a_{1}, a_{2}$ account for mass and mechanical resistance. And $r(v)$ has following characteristics:

$$
r^{\prime}(v)>0, \quad r^{\prime \prime}(v) \geq 0
$$

The energy efficient operation problem is modeled as follows:

$$
\begin{aligned}
\min J & =\int_{0}^{X} u_{f} f(v) d x \\
\text { s.t. } 0 & \leq u_{f} \leq 1,0 \leq u_{b} \leq 1, \\
v & \leq V(x)
\end{aligned}
$$

where $J$ is the specific mechanical work of the traction force, $V(x)$ is the speed limit. The boundary conditions are

$$
\begin{array}{ll}
x(0)=x_{0}, & x(T)=x_{T}, \\
v(0)=v_{0}, & v(T)=v_{T} .
\end{array}
$$

By applying Pontryagin maximum principle to solve the problem as specified in (15) with constraints (16), the optimal solution should maximize the Hamiltonian function:

$$
\begin{aligned}
H= & \frac{p_{1}}{v}\left(u_{f} f(v)-u_{b} b(v)-r(v)\right) \\
& +\frac{p_{2}}{v}-u_{f} f(v),
\end{aligned}
$$

where $p_{1}$ should satisfy the differential equation:

$$
\frac{d p_{1}}{d x}=-\frac{\partial H}{\partial v} .
$$

It is easy to prove that the Hamiltonian reaches the maximum with respect to $u_{f}$ and $u_{b}$. And there are five energy efficient control modes as follows.

(i) Full power $\left(u_{f}=1, u_{b}=0\right)$ if $\left(p_{1} / v\right)<0$.

(ii) Partial power $\left(u_{f} \in(0,1), u_{b}=0\right)$ if $\left(p_{1} / v\right)=0$.

(iii) No power and no braking $\left(u_{f}=0, u_{b}=0\right)$ if $0<$ $\left(p_{1} / v\right)<1$.

(iv) Partial braking $\left(u_{f}=0, u_{b} \in(0,1)\right)$ if $\left(p_{1} / v\right)=1$.

(v) Full braking $\left(u_{f}=0, u_{b}=1\right)$ if $\left(p_{1} / v\right)>1$.
They could be seen as four possible driving phases, which are

(i) acceleration with full power;

(ii) speed holding with partial power or braking;

(iii) coasting with no power and braking;

(iv) braking with full braking.

Based on the above analysis, it is seen that the energy saving strategy relies on these optimal controls. Train energy saving driving process is the process of switching from an optimal control to another, and the switching sequence is acceleration, speed holding, coasting, and braking.

Then, the optimal control switching speeds and running phases duration are treated as decision parameters. The energy and power can be expressed by these decision parameters. The power demand is used as the objective function of a nonlinear programming problem. The constraints include running time and distance. In actual train operation, maximum power and braking cannot be applied by considering the ride comfort. Instead, a service acceleration/braking rate is applied. Therefore, by solving the nonlinear programming model, a reference trajectory leading to less power and energy consumption can be obtained.

\section{Peak Demand Reduction Techniques}

3.1. Service Headway Braking Strategy. Based on the analysis above, we know the restarting of the dense queue in a small area leads to peak power demand and both of the two traditional PDR techniques are carried out after the formation of the dense queue. In this section, we propose a novel operation strategy to reduce the peak power by avoiding the formation of a dense queue based on the available extra station dwell time. In the following parts, we use reaccelerate to indicate train accelerates from a nonzero initial speed and restart to indicate train starts from $0 \mathrm{~m} / \mathrm{s}$.

3.1.1. SHB Strategy Analysis. Figure 3 shows the new operation strategy; the leading train stops at station $\mathrm{A}$ and the position is $S_{1}$. The black thick solid line is calculated by (2), and we call it braking curve in this paper. Under normal circumstance, the following train should brake when it touches the brake curve. However, if the leading train starts to run when the following train touches braking curve, the following train will not brake but reaccelerate. Therefore, in the new strategy, if the following train touches the braking curve when the leading train starts to run, and the speed at this moment is not $0 \mathrm{~m} / \mathrm{s}$, the moment in which each train reaches the peak power demand can be staggered. Meanwhile, since the following trains brake at first, they will not stop too to cause a dense queue. By these two reasons, the total peak power demand can be reduced. Based on the analysis above and considered the energy saving operation process, a new operation strategy is proposed as follows.

As shown in Figure 2(a), the target speed of the trains in the track is $v_{1}$; the leading train has an extra dwell time, which is $T_{\text {delay. }}$. If $T_{\text {delay }}$ is long enough to cause a dense queue with $n$ trains, then, after $T_{\mathrm{dwell}}$, let train $i(i=2,3, \ldots, n)$ brake with service braking deceleration $b$ to $v_{i 2}$ (the position at this time 


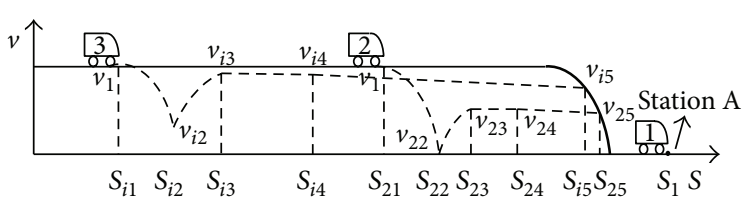

(a)

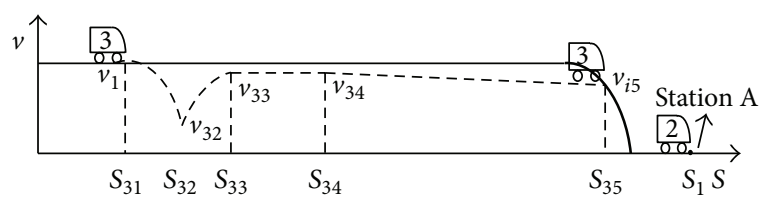

(b)

FIgure 2: (a) Following trains' SHB driving curves after train 1 stops for $T_{\text {dwell }}$. (b) The position of train 3 when train 2 starts to move.

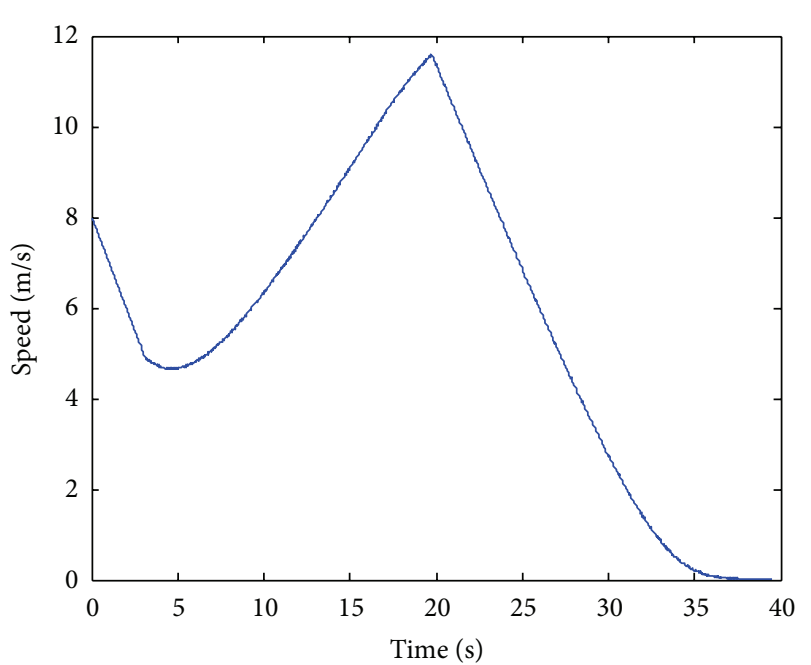

FIGURE 3: $V$-T profile of the following train starts form the braking profile.

is $\left.S_{i 2}\right)$; if $v_{i 2}=0$, then it stops at $S_{i 2}$ for $\Delta t_{i 2}$, then accelerates to $v_{i 3}$ with service acceleration $a$ (the position at this time is $\left.S_{i 3}\right)$, and then runs with $v_{i 4},\left(v_{i 4}=v_{i 3}\right)$ for $\Delta t_{i 4}$ to $S_{i 4}$; after that it reduces traction force and keeps the train moving with a constant deceleration $b^{\prime}$ to $v_{i 5}$ (the position at this time is $S_{i 5}, v_{i 5}$ and $S_{i 5}$ are a pair of points in the braking curve). At this time, train $i-1$ restarts, therefore, train istarts to track train $i-1$ according to the moving block tracking distance interval until arrives at station A.

Figure 2(b) shows the position of train 3 when train 2 starts to move from station A. It is observed that train 3 will reaccelerate from the braking curve $\left(S_{35}\right)$ to arrive at station $\mathrm{A}\left(S_{1}\right)$; we use $T_{t t}$ to represent this time period and all of the delayed following trains need this time period to arrive at station A after their leading train's restarting. In this circumstance, for train 2, the running time between $S_{21}$ and $S_{25}$ is $T_{\text {delay }}$; for train 3, the running time between $S_{31}$ and $S_{35}$ is $T_{\text {delay }}+T_{\text {dwell }}+T_{t t} ; \ldots$; for train $i$, the running time between $S_{i 1}$ and $S_{i 5}$ is $T_{\text {delay }}+(i-2) \times\left(T_{\text {dwell }}+T_{t t}\right)$.

In order to obtain $T_{t t}$, a simulation is done as follows: $L_{t}=140(\mathrm{~m}), L_{\text {safe }}=50(\mathrm{~m})$. The leading train stops at station A where the position is $400(\mathrm{~m}): S_{\text {leading }}(0)=400(\mathrm{~m})$. Let the following train start from the point in braking curve; the speed and position of the following train is calculated by (4) or (5); the results are shown in Table 1.

From Table 1, we can see that the arrival times of following trains seem constant when they reaccelerate from the braking curve. In order to show the trend of this tracing process, Figure 3 gives the $V-T$ profile when the reaccelerating speed and position are $8 \mathrm{~m} / \mathrm{s}$ and $178(\mathrm{~m})$.
TABLE 1: Running time of following train.

\begin{tabular}{lccc}
\hline $\begin{array}{l}S_{\text {leading }}(0) \\
(\mathrm{m})\end{array}$ & $\begin{array}{c}V_{\text {following }}(0) \\
(\mathrm{m} / \mathrm{s})\end{array}$ & $\begin{array}{c}S_{\text {following }}(0) \\
(\mathrm{m})\end{array}$ & $\begin{array}{c}\text { Running time of the } \\
\text { following train arrives at } \\
\text { station A } \\
(\mathrm{s})\end{array}$ \\
\hline 400 & 16 & 82 & 40.26 \\
400 & 12 & 138 & 39.82 \\
400 & 8 & 178 & 39.5 \\
400 & 4 & 202 & 39.42 \\
400 & 0 & 210 & 39.38 \\
\hline
\end{tabular}

Based on the analysis above, the whole strategy is consisting of 6 steps, braking, waiting, traction, speed holding, coasting, and finally tracking the front train. The indexes of them are $1,2, \ldots, 6$.

It is worth noting that this operation strategy is energy efficient, because the duration of some steps may be 0 based on an appropriate target function. For example, if we get $v_{i 2}=$ $v_{i 3}=v_{i 4}>0$ based on a certain target function, there will be no traction process before reaccelerating and the train will brake and coast until reaccelerating.

In this new strategy, all the following trains brake at first; therefore, they will not stop too to cause a dense queue; at the same time, the other trains are coasting when one train reaccelerates, so the reaccelerating times of the following trains are staggered. Therefore the peak power demand is avoided.

3.1.2. Model of SHB. In this section, we formulate the mathematical model of the operation process. For train $i$, define $t_{i j}(j=1,2, \ldots, 5)$ as the starting time of each step; $\Delta S_{i j}(j=$ $1,2, \ldots, 5)$ and $\Delta T_{i j}(j=1,2, \ldots, 5)$ are the running distance and time of each step, respectively. In order to stagger the reaccelerating times of the following trains, we should take an appropriate value to $v_{i 5}$. Because if $v_{i 5}=0$, the successive two trains will reaccelerate simultaneously again and peak power demand cannot be reduced. On the contrary, if $v_{i 5}$ is close to $v_{1}$, the effect of energy saving will be reduced. Therefore, we recommend $v_{1}>v_{i 5} \geq v_{1} / 2$. At the same time, for train $i$, the total running time and distance should satisfy the following equations:

$$
\begin{gathered}
\sum_{j=1}^{5} \Delta T_{i j}=T_{\text {delay }}+(i-2) \times\left(T_{\text {dwell }}+T_{t t}\right), \\
\sum_{j=1}^{5} \Delta S_{i j}=S_{1}-S_{i 1}-L_{\text {safe }}-L_{t} .
\end{gathered}
$$


Since the traction phase is highly energy consumed and power supported, we minimize the peak power. Therefore, based on the analysis above, the problem could be seen as a constrained nonlinear programming problem as follows:

$$
\min f=\sum_{i=1}^{n} p_{i}\left(v_{i 3}\right)
$$

$$
\begin{array}{ll}
\text { s.t. } & v_{i 2}-v_{i 3} \leq 0, \\
& v_{i 5}-v_{i 4} \leq 0, \\
& -v_{i j},-\Delta T_{i j} \leq 0, \\
& \frac{v_{1}}{2} \leq v_{i 5}<v_{1}, \\
& \sum_{j=1}^{5} \Delta T_{i j}=T_{\text {delay }}+(i-2) \times\left(T_{\text {dwell }}+T_{t t}\right), \\
\sum_{j=1}^{5} \Delta S_{i j}=S_{1}-S_{i 1}-L_{\text {safe }}-L_{t},
\end{array}
$$

where

$$
\begin{aligned}
\sum_{j=1}^{5} \Delta T_{i j}= & \frac{v_{i 2}-v_{1}}{b}+\Delta T_{i 2}+\frac{v_{i 3}-v_{i 2}}{a} \\
& +\Delta T_{i 4}+\frac{v_{i 5}-v_{i 4}}{b^{\prime}}, \\
\sum_{j=1}^{5} \Delta S_{i j}+L_{b}= & \frac{v_{i 2}^{2}-v_{1}^{2}}{2 \times b}+\frac{v_{i 3}^{2}-v_{i 2}^{2}}{2 \times a} \\
& +\Delta T_{i 4} \times v_{i 4}+\frac{v_{i 5}^{2}-v_{i 4}^{2}}{2 \times b^{\prime}}+\frac{v_{i 5}^{2}}{2 \times|b|},
\end{aligned}
$$

where $\alpha, \beta$ are penalty factors, $\alpha, \beta>0 ; a, b$ are service acceleration and deceleration of the train; $b^{\prime}$ is a very small deceleration; $i$ is the index of the delayed following trains $i=2, \ldots, n ; j$ is the index of operation steps, $j=1,2, \ldots, 6 ; v_{i j}$ is the starting speed of each step for train $i ; S_{1}$ is the position of the leading train (the leading train); $S_{i 1}$ is the position of the $i$ th following train; $\Delta S_{i j}$ is the running distance of the $j$ th step for train $i ; \Delta T_{i j}$ is the duration of of the $j$ th step for train $i$; $L_{i b}$ is the braking distance if train $i$ brakes from $v_{i 5} ; T_{\text {dwell }}$ is the scheduled station dwell time; $T_{t t}$ is the tracking time in which train $i$ tracks train $i-1$ from $S_{i 5}$ to $S_{1}$.

During the running process of successive following trains, the time interval among them is $T_{\mathrm{dwell}}+T_{t t}$. When train $i$ starts to move from $S_{i 2}$, the distance between train $i$ and train $i-1$ is shortest; at this time, the speed of train $i$ is larger than $v_{1} / 2$ and the distance interval between them is at least $\left(T_{\text {dwell }}+\right.$ $\left.T_{t t}\right) \times v_{1} / 2$; according to the general condition in mass transit system, $T_{\text {dwell }}=10 \mathrm{~s}, L_{\text {safe }}=50 \mathrm{~m}, L_{t}=140 \mathrm{~m}, \operatorname{MAX}\left(v_{1}\right)=$ $20 \mathrm{~m} / \mathrm{s}, b=1 \mathrm{~m} / \mathrm{s}^{2}$, it is easy to know that $\left(T_{\text {dwell }}+T_{t t}\right) \times v_{1} / 2>$ $L_{\text {safe }}+L_{t}+\left(v_{1} / 2\right)^{2} / 2 b$; this is satisfied by (3). Therefore, it is no need to consider the distance constraint shown in (3) between successive following trains in (22) again.

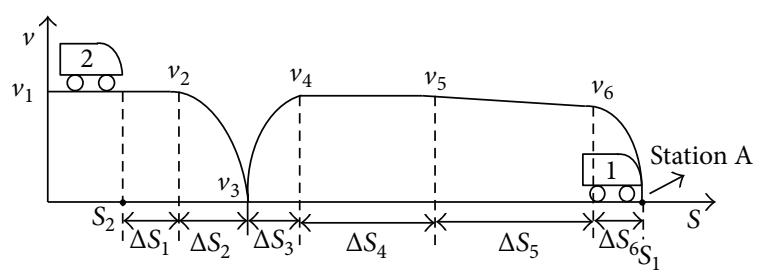

FIgURE 4: Operation strategy of ESDI.

3.2. Extending Stopping Distance Interval Strategy. The previous section shows SHB strategy; it could reduce the peak power demand if the delay time is available. In this section, we will show a strategy which could reduce the peak power demand by decreasing the density of the dense queue. By adopting this strategy, the peak power demand could be reduced if the extra station dwell time is unavailable. Because, in the same $T_{\text {delay, }}$, the longer the stopping distance interval of the following trains, the lower densities of the stopped train queue. Therefore, when they restart, the peak power demands are reduced.

3.2.1. ESDI Strategy Analysis. According to the analysis in Section 3.1.1, it can be seen that no matter the train reaccelerates from which point in the braking curve, $T_{t t}$ is nearly the same. Based on $T_{t t}$, we can extend the stopping distance interval of the following trains to reduce the density of the dense queue. The new operation strategy is proposed as follows.

Figure 4 shows the new operation strategy. The operation process of the following trains when the leading train dose does not move after $T_{\text {dwell }}$ could be divided into 6 steps, speed holding, braking, traction, speed holding, coasting, and braking to stop. Define $t_{i}(i=1,2 \ldots, 6)$ as the starting time of each step, $v_{1}=v_{2}$, and $v_{3}=0, v_{4}=v_{5}$; define the running time and distance of each step as $\Delta T_{i}$ and $\Delta S_{i}(i=$ $1,2, \ldots, 6)$, respectively. When the leading train has an extra dwell time, each of following trains will run according to these 6 steps. Define $M I$ as the traveling distance during $T_{t t}(M I$ is also stopping distance interval) for the following trains. During this process, the running time of steps 3 to 6 should be equal to $T_{t t}$.

In this new strategy, the stopping distance interval of the following trains is increased, so the density of the queue is decreased, and the peak power demand is reduced.

3.2.2. Model of ESDI. In this section, we formulate the mathematic model of the operation process. According to the last section, it is known that we know that the stopping intervals of the trains are extended in the new operation strategy; therefore, peak power demand can be reduced when the trains restart. Define $M I$ as the stopping interval of two successive trains; we have

$$
\begin{aligned}
M I & =\sum_{i=3}^{6} \Delta S_{i} \\
& =\frac{v_{4}^{2}}{2 \times a}+v_{4} \times \Delta T_{4}+\frac{\left(v_{6}^{2}-v_{5}^{2}\right)}{2 \times b^{\prime}}+\frac{v_{6}^{2}}{2 \times|b|} .
\end{aligned}
$$


In order to grantee the feasibility of the new operation strategy, if leading train stops at the station, after $T_{\text {dwell }}$, the distance between the first following and the leading train should satisfy the following equation:

$$
M I \leq S_{\text {tracking }}-T_{\text {dwell }} \times v_{1},
$$

where $S_{\text {tracking }}$ is the second train's position when the leading train stops at station, and it is calculated by

$$
S_{\text {tracking }}=\left(T_{\text {traking }}-\frac{v_{1}}{|b|}\right) \times v_{1}+\frac{v_{1}^{2}}{2 \times|b|} .
$$

Since the traction phase is highly energy consumed and power supported, we minimize the peak power. Based on the analysis above, we use nonlinear programming to model the problem as follows:

$$
\begin{aligned}
& \min f=p\left(v_{4}\right), \\
& \text { s.t. } v_{3}-v_{2} \leq 0, \\
& \quad v_{6}-v_{5} \leq 0, \\
& M I \leq S_{\text {tracking }}-T_{\text {dwell }} \times v_{1}, \\
& \quad\left(T_{\text {dwell }}+\Delta T_{1}\right) \times v_{1}+\frac{v_{1}^{2}}{2 \times|b|}+\sum_{i=3}^{6} \Delta S_{i}=S_{2}-S_{1}, \\
& \quad \sum_{i=3}^{6} \Delta T_{i}-T_{t t}=0,
\end{aligned}
$$

where $\alpha, \beta$ are penalty factors, $\alpha>0 ; a, b$ are service acceleration and deceleration of the train; $v_{i}$ is the starting speed of step $i ; S_{1}$ is the position of the leading train (the leading train); $S_{2}$ is the position of the following train; $S_{\text {tracking }}$ is the second train's position when the leading train stops at station; $T_{\text {dwell }}$ is the scheduled station dwell time.

\section{Simulation and Discussion}

In this section, a simulation is used to test and verify the new strategies. The length of train $\left(L_{t}\right)$ is $140 \mathrm{~m}$, safety margin $\left(L_{\text {safe }}\right)$ is $50 \mathrm{~m}$, service tracking headway is 120 seconds, dwell time $\left(T_{\text {dwell }}\right)$ is 10 seconds, target speed $\left(v_{1}\right)$ is $16 \mathrm{~m} / \mathrm{s}$, service acceleration rate $(a)$ is $1 \mathrm{~m} / \mathrm{s}^{2}$, service braking deceleration rate $(b)$ is $1 \mathrm{~m} / \mathrm{s}^{2}$, and position of station $\mathrm{A}$ is $3710 \mathrm{~m}\left(S_{1}=\right.$ $3710)$.

In order to choose $b^{\prime}$, we analyze the practical data of coasting phase from Dalian Fast Track [24]. Because the speed in coasting phase declines very slowly, so the profile of speed time could be seen as a straight line and the slope of the line is the deceleration rate of coasting phase. We use leastsquare procedure to fit the speed-time date sectional and the results are shown in Table 2.

From Table 2, it is clear that the higher the coasting starting speed, the greater the deceleration. In order to supply a small traction force to keep the train moving in a constant deceleration $b^{\prime}$, we choose $b^{\prime}=0.01 \mathrm{~m} / \mathrm{s}^{2}$ as appropriate.
TABLE 2: Measured value of coasting data.

\begin{tabular}{lcc}
\hline Speed range $(\mathrm{km} / \mathrm{h})$ & Slop $\left(\mathrm{m} / \mathrm{s}^{2}\right)$ & Average error $(\mathrm{m})$ \\
\hline $37-31$ & -0.0147 & 0.0227 \\
$41-40$ & -0.0125 & 0.0361 \\
$43-42$ & -0.0147 & 0.0364 \\
$54-48$ & -0.0213 & 0.0236 \\
$60-59$ & -0.0237 & 0.0296 \\
$62-61$ & -0.0210 & 0.0301 \\
$79-75$ & -0.0315 & 0.0237 \\
\hline
\end{tabular}

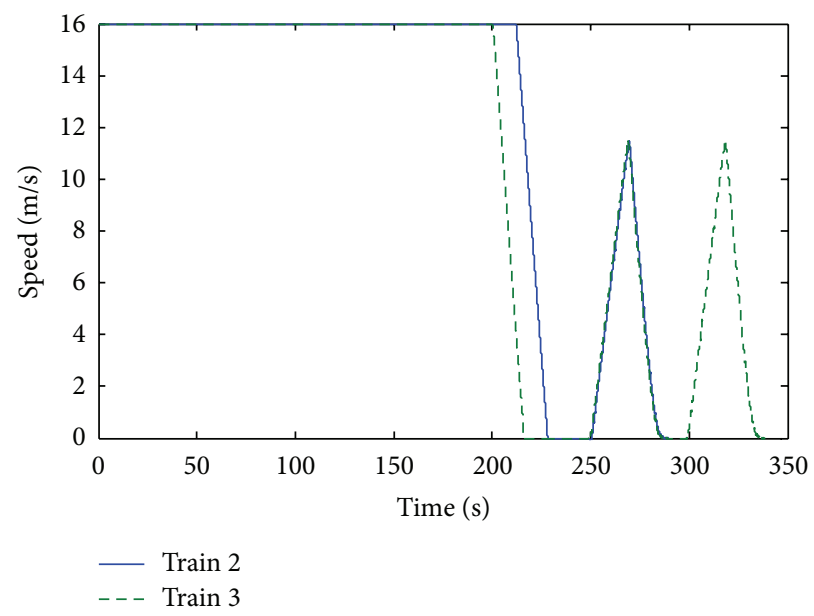

Figure 5: $V$-T profile without PDR technique.

4.1. Service Headway Braking Strategy. If $T_{\text {delay }}$ is 250 seconds, according to (8), 3 trains will be delayed (including the leading train). Because we choose $v_{\text {ref }}=8$, therefore, $T_{t t}=$ 38.5 is selected from Table 1. Based on (21)-(22), we have

$$
\begin{gathered}
v_{22}=0, \quad v_{23}=9.57, \quad v_{25}=8, \quad \Delta t_{24}=0, \\
v_{32}=13.043, \quad v_{33}=13.043, \\
v_{35}=10.08, \quad \Delta t_{34}=0 .
\end{gathered}
$$

Figures 9 and 10 and Table 3 show the simulation results.

In order to show the peak power demand without any PDR technique and compare it with the performance of traditional PDR technique, Figures 5-8 are given. According to Figures 5 and 6 , we can see that the two following trains are starting simultaneously from $250 \mathrm{~s}$; the peak power demand is $25.1 \mathrm{kw} / \mathrm{t}$. The arrival times of train 2 and train 3 are $289.38 \mathrm{~s}$ and $338.76 \mathrm{~s}$, respectively.

Figures 7 and 8 show the performance of graded ARL technique. The acceleration of train 2 and train 3 is $0.5 \mathrm{~m} / \mathrm{s}^{2}$ and $0.3 \mathrm{~m} / \mathrm{s}^{2}$. By applying different acceleration, the peak power demand is reduced to $22.07 \mathrm{kw} / \mathrm{t}$. However, the time delay is increased. The arrival times of train 2 and train 3 are $297.78 \mathrm{~s}$ and $400.36 \mathrm{~s}$, respectively. That means the arrival times of the two trains are $8.4 \mathrm{~s}$ and $61.6 \mathrm{~s}$ later than their times without PDR techniques, respectively.

The performance of applying SHB technique is shown from Figures 9 and 10. As can be seen, train 2 has a waiting 
TABLE 3: Comparison of the graded ARL and SHB techniques.

\begin{tabular}{|c|c|c|c|c|c|c|}
\hline & \multirow{2}{*}{$\begin{array}{l}\text { Arrival time of } \\
\text { train } 2(\mathrm{~s})\end{array}$} & \multirow{2}{*}{$\begin{array}{l}\text { Arrival time } \\
\text { of train } 3 \text { (s) }\end{array}$} & \multirow{2}{*}{$\begin{array}{c}\text { Peak power } \\
\text { demand }(\mathrm{kw} / \mathrm{t})\end{array}$} & \multirow{2}{*}{$\begin{array}{c}\text { Energy } \\
\text { consumption }(\mathrm{kw} \cdot \mathrm{h})\end{array}$} & \multicolumn{2}{|c|}{ Stopping time before arrival (s) } \\
\hline & & & & & Train 2 & Train 3 \\
\hline Non-PDR & 289.38 & 338.76 & 25.1 & 15.9137 & 130 & 43.75 \\
\hline $\begin{array}{l}\text { Graded } \\
\text { ARL }\end{array}$ & 297.78 & 400.36 & 22.07 & 46.3402 & 130 & 43.75 \\
\hline SHB & 289.5 & 339.16 & 13.42 & 10.5016 & 65.66 & 0 \\
\hline
\end{tabular}

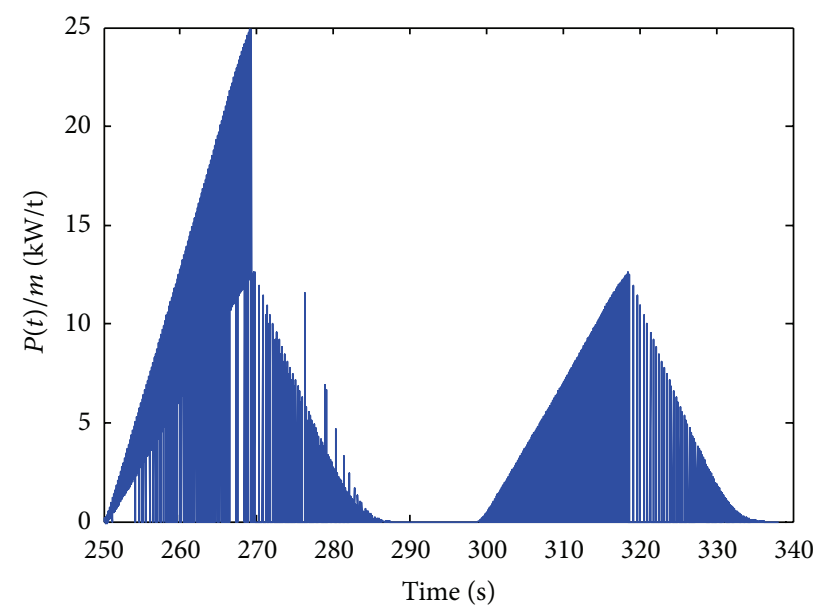

FIGURE 6: Peak power demand profile without PDR technique.

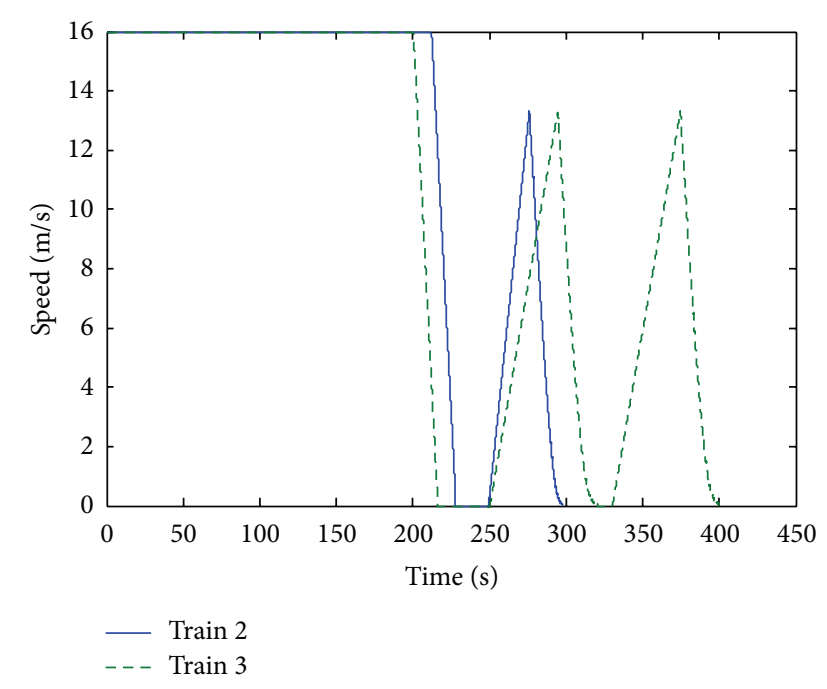

FIGURE 7: $V$-T profile with graded ARL technique.

time $65.66 \mathrm{~s}$ at the position of $2048 \mathrm{~m}$ and then restarting at $81.66 \mathrm{~s}$. At this time, train 3 is coasting; therefore, there is no peak power in this time. The two trains restart with different speeds in different time points, which can be seen form the red and blue circles in Figure 9. That means the reaccelerating times of the two trains are staggered. Furthermore, the two trains reaccelerate with nonzero initial speeds, which means the reacceleration periods are reduced. Therefore, the peak power demand is reduced to $13.42 \mathrm{kw} / \mathrm{t}$. According to Figure 9, the two trains have no speed holding phases, and

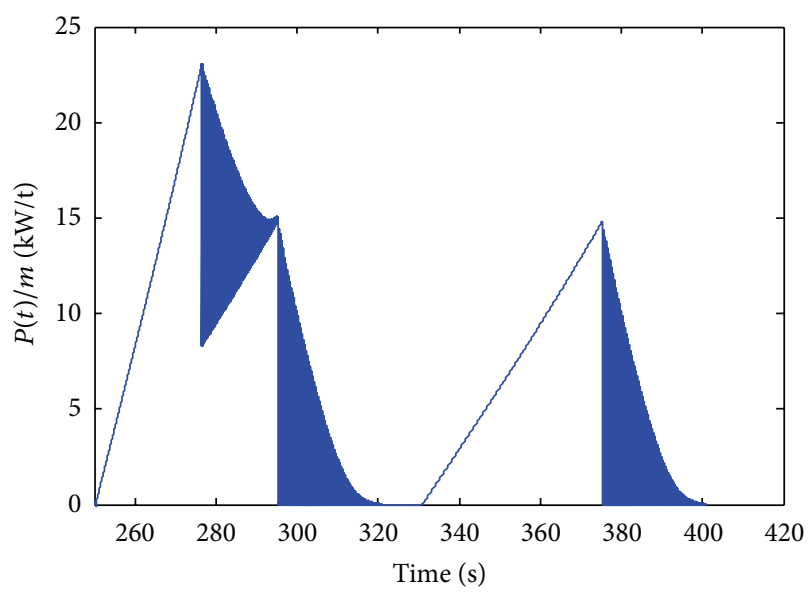

FIGURE 8: Peak power demand profile with graded ARL technique.

train 3 has no traction phases before step 6 . Therefore, the energy consumption is reduced. The arrival times of train 2 and train 3 are $289.5 \mathrm{~s}$ and $339.16 \mathrm{~s}$, respectively.

Table 3 shows the result data of the simulation. Graded ARL technique can reduce the peak power demand; however, the arrival times of the two trains are delayed significantly. SHB technique has great advantages. According to Table 3, the delay times of the two trains are very short; at the same time, the energy consumption is also reduced to a low level, even less than value without any PDR technique. In addition, the stopping times before arrival of the two trains are reduced to 65.66 and 0 , respectively.

4.2. Extending Stopping Distance Interval Strategy. According to Section 3.1.2, we choose $T_{t t}=40 \mathrm{~s}$; when the leading train stops at the station and dose not start to run after $T_{\text {dwell }}$, based on (27), we have

$$
v_{4}=v_{5}=16, \quad v_{6}=15.92, \quad \Delta T_{4}=0 .
$$

If the extra dwell time is 250 seconds, in graded ARL, 3 trains will be delayed (including the leading train) according to (8). And the length of the dense queue is $380 \mathrm{~m}$. The performance of graded ARL technique can be seen in Figure 5.

In ESDI, based on (24) the stopping distance interval for the following trains $(M I)$ is $383.68 \mathrm{~m}$. Based on (8), 2 trains (besides the leading train) will be delayed. However, the length of the dense queue is $767.36 \mathrm{~m}$. In the same time period and distance area with graded ARL technique, we calculate the peak power demand, and the performance is shown in Figures 11 and 12 . 

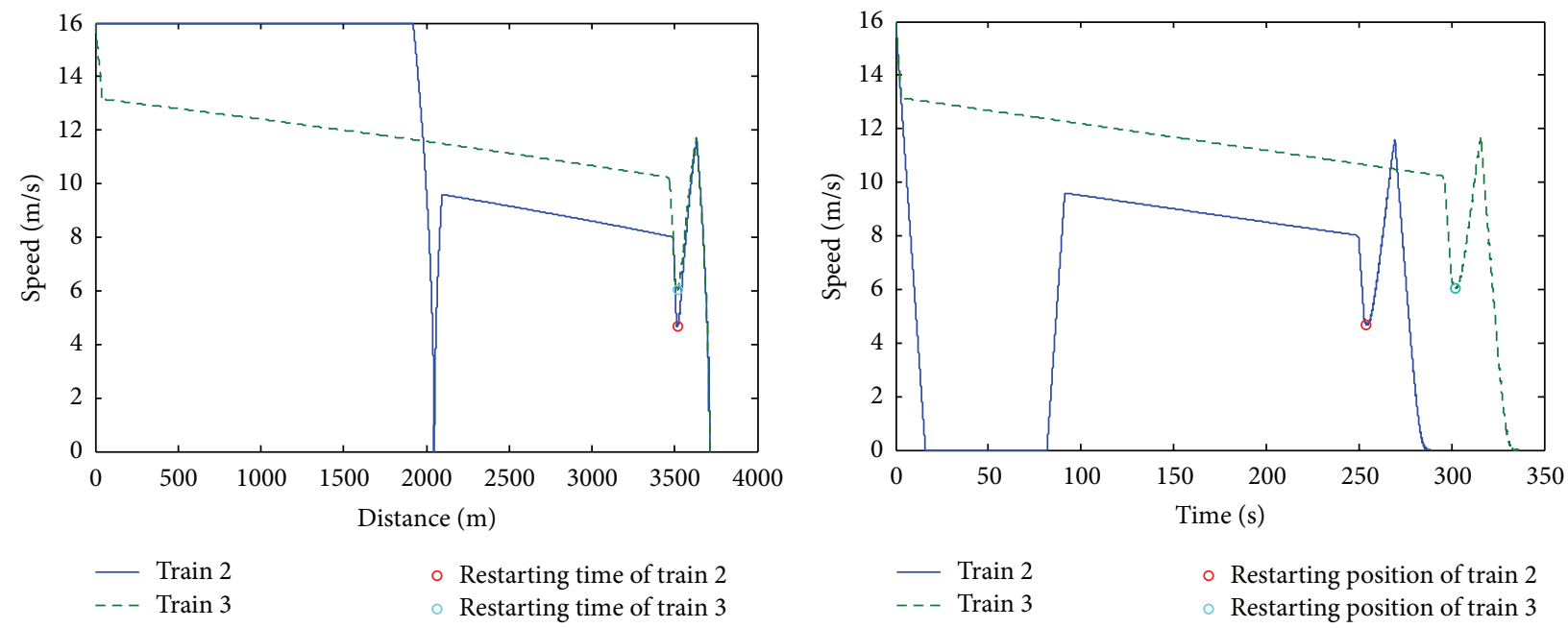

Figure 9: $V-S$ and $V-T$ profiles with SHB technique.

TABLE 4: Comparison the PDR techniques.

\begin{tabular}{|c|c|c|c|c|c|c|}
\hline & \multirow{2}{*}{$\begin{array}{l}\text { Arrival time } \\
\text { of train } 2(\mathrm{~s})\end{array}$} & \multirow{2}{*}{$\begin{array}{l}\text { Arrival time } \\
\text { of train } 3(\mathrm{~s})\end{array}$} & \multirow{2}{*}{$\begin{array}{l}\text { Peak power } \\
\text { demand (kw/t) }\end{array}$} & \multirow{2}{*}{$\begin{array}{l}\text { Energy } \\
\text { consumption }(\mathrm{kw} \cdot \mathrm{h})\end{array}$} & \multicolumn{2}{|c|}{ Stopping time before arrival (s) } \\
\hline & & & & & Train 2 & Train 3 \\
\hline Non-PDR & 289.38 & 338.76 & 25.1 & 15.9137 & 130 & 43.75 \\
\hline $\begin{array}{l}\text { Graded } \\
\text { ARL }\end{array}$ & 297.78 & 400.36 & 22.07 & 46.3402 & 130 & 43.75 \\
\hline SHB & 289.5 & 339.16 & 13.42 & 10.5016 & 65.66 & 0 \\
\hline ESDI & 290 & 340 & 18.18 & 22.86 & 153.98 & 57.96 \\
\hline
\end{tabular}

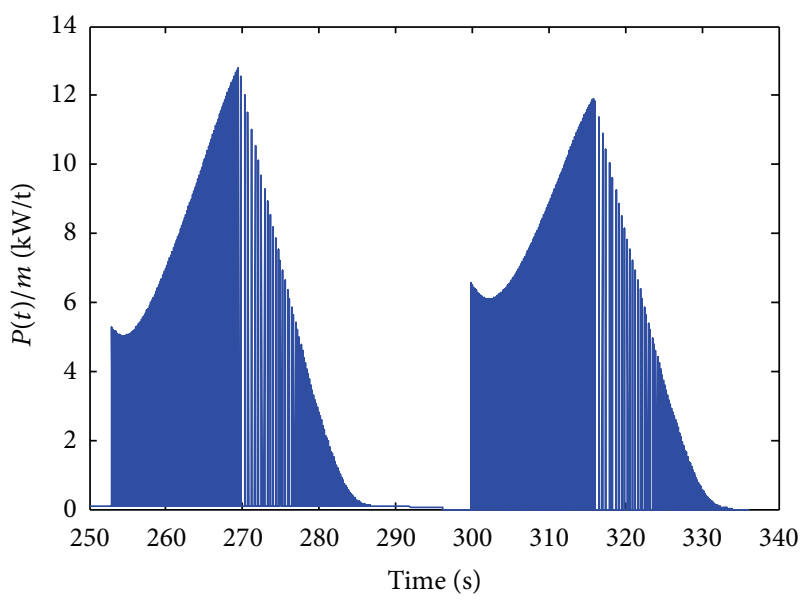

FIGURE 10: Peak power demand profile with SHB technique.

As we see, using ESDI technique, the arrival times of the following two trains are almost the same as their times with no PDR technique. Without any PDR technique, the stopping distance interval is $190 \mathrm{~m}$, so the dense queue caused by the extra station time $(250 \mathrm{~s})$ is $380 \mathrm{~m}$. The peak power demand after $250 \mathrm{~s}$ within $380 \mathrm{~m}$ is $25.1 \mathrm{kw} / \mathrm{t}$. In ESDI strategy, stopping distance interval is extended to $383.86 \mathrm{~m}$, which is two times longer than the distance in ARL. That is to say, the restarting position of train 2 belongs to the nearby substation. Thus the power demands by train 2 and train 3 are not afforded by the same substation. Therefore, the peak power demand after $250 \mathrm{~s}$ within $380 \mathrm{~m}$ is reduced to $18.18 \mathrm{kw} / \mathrm{t}$. Compared with non-PDR technique, the peak power demand is reduced by $18 \%$. In ESDI, train 2 has a stopping time $153.98 \mathrm{~s}$ at the position of $2944.6 \mathrm{~m}$. Train 3 has a stopping time $57.96 \mathrm{~s}$ at the position of $3328.32 \mathrm{~m}$. Compared with nonPDR technique, the stopping time before arrival is increased by $18 \%$.

According to above analysis, we can see that the new strategy reduces peak power demand by sacrificing the stopping time before arrival. However, the advantages of ESDI are still obvious, because it is more efficient on energy saving; it can reduce energy consumption by $50 \%$ compared with graded ARL technique.

From Table 4, it is seen that, although the arrival times of trains when applying SHB and the arrival times of trains when applying ESDI are the same, SHB reduces more peak power demand and energy consumption, which means SHB performs than ESDI when the available and unavailable extra station dwell times are the same.

\section{Conclusion}

Peak power demand reduction strategies are discussed in this paper. The reasons of peak power demand problem are analyzed deeply and two main reasons are given. 

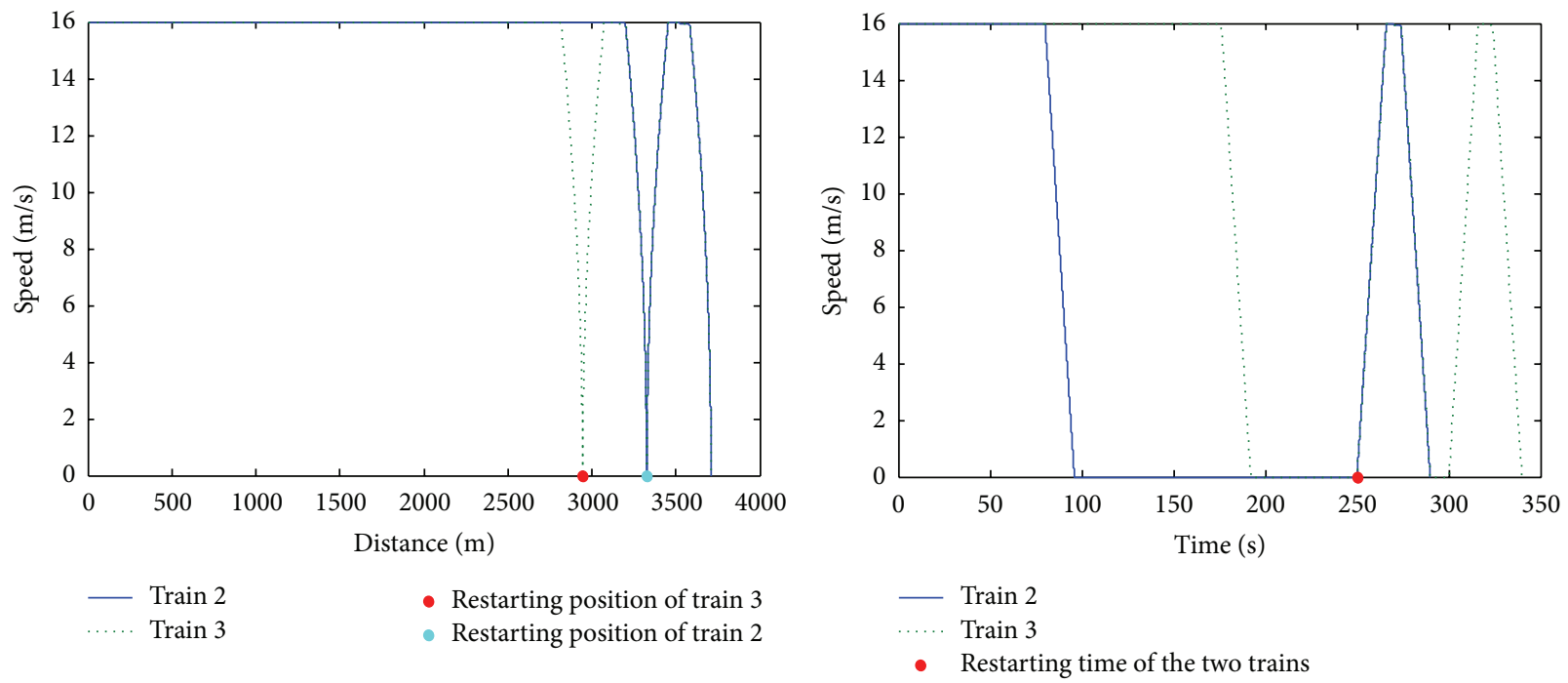

FIgURE 11: $V$-S and $V$-T profiles with ESDI technique.

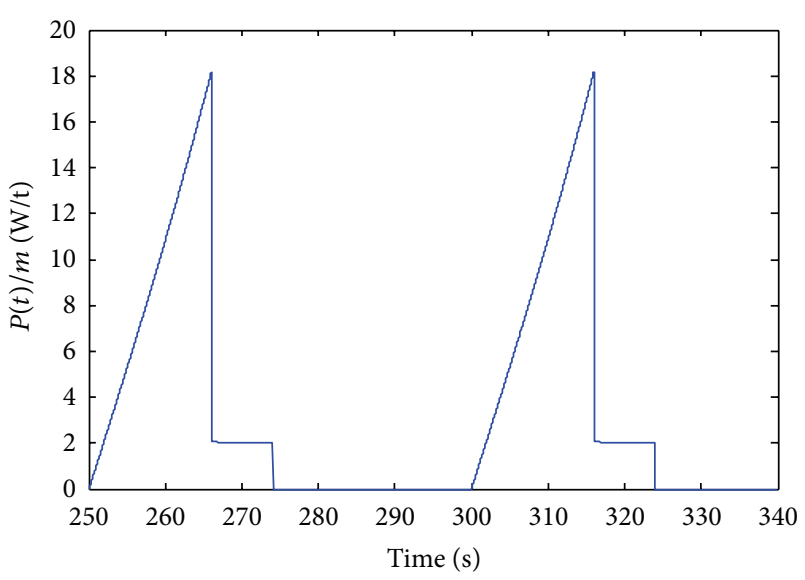

FIGURE 12: Peak power demand profile with ESDI technique.

Based on the reasons and according to different situations, two new peak demand reduction techniques are proposed. One is Service Headway Braking (SHB) strategy, which is used to reduce peak power demand when the extra station dwell time is available. The other is Extending Stopping Distance Interval (ESDI) strategy, which is used to reduce peak power demand when the extra station dwell time is unavailable. Nonlinear programming approach is introduced to model the operation strategy. The simulation results show that, compared with the best traditional PDR techniques, SHB can reduce $40 \%$ of peak power demand and $77 \%$ of energy consumption without increasing the arrival time delay. ESDI can reduce $20 \%$ of peak power demand and $50 \%$ of energy consumption without increasing the arrival time delay. Therefore, SHB has a better performance than ESDI when the available and unavailable extra station dwell times are the same. The basic principle of the two strategies is to avoid the formation of the dense queue. Therefore, both of the two strategies are implemented before the formulation of the dense queue.

\section{Conflict of Interests}

The authors declare that there is no conflict of interests.

\section{Acknowledgments}

This work was supported by the National High Technology Research and Development Program of China (no. 2011AA110502) and the National Natural Science Foundation of China (no. 61134001).

\section{References}

[1] L. V. Pearson, Moving block railway signalling [Ph.D. thesis], Loughborough University of Technology, Loughborough, UK, 1973.

[2] T. Chaiyatham and I. Ngamroo, "Optimal fuzzy gain scheduling of PID controller of superconducting magnetic energy storage for power system stabilization," International Journal of Innovative Computing, Information and Control, vol. 9, no. 2, pp. 651666, 2013.

[3] D. Iannuzzi, E. Pagano, and P. Tricoli, "The use of energy storage systems for supporting the voltage needs of urban and suburban railway contact lines," Energies, vol. 6, pp. 1802-1820, 2013.

[4] H. Lee, S. Jung, Y. Cho, H. Jung, H. Kim, and G. Jang, "Peak power reduction and energy efficiency improvement with the superconducting flywheel energy storage in electric railway system," Physica C, vol. 494, pp. 246-249, 2013.

[5] Y. Arai, H. Seino, K. Yoshizawa, and K. Nagashima, "Development of superconducting magnetic bearing with superconducting coil and bulk superconductor for flywheel energy storage system," Physica C, vol. 494, pp. 250-254, 2013.

[6] H. Takeuchi, C. J. Goodman, and S. Sone, "Peak demand reduction techniques when starting under Moving Block Signalling," 
in Proceedings of the International Conference on Developments in Mass Transit Systems, pp. 280-285, April 1998.

[7] H. Takeuchi and C. J. Goodman, "Simulation study of peak demand reduction strategies when starting under moving block signalling," in Proceedings of the 5th International Conference on Computer Aided Design, Manufacture and Operation in the Railway and other Advanced Mass Transit Systems, pp. 187-196, August 1996.

[8] H. Takeuchi, C. J. Goodman, and S. Sone, "Moving block signalling dynamics: performance measures and re-starting queued electric trains," IEE Proceedings, vol. 150, no. 4, pp. 483-492, 2003.

[9] T. K. Ho and K. K. Wong, "Peak power demand reduction under moving block signalling using an expert system," IEE Proceedings, vol. 150, no. 4, pp. 471-482, 2003.

[10] T. Albrecht, "Reducing power peaks and energy consumption in rail transit systems by simultaneous train running time control," Advances in Transport, vol. 15, pp. 885-894, 2004.

[11] J.-F. Chen, R.-L. Lin, and Y.-C. Liu, "Optimization of an MRT train schedule: reducing maximum traction power by using genetic algorithms," IEEE Transactions on Power Systems, vol. 20, no. 3, pp. 1366-1372, 2005.

[12] K. M. Kim, S.-M. Oh, and M. Han, "A mathematical approach for reducing the maximum traction energy: the case of Korean MRT trains," in Proceedings of the International MultiConference of Engineers and Computer Scientists (IMECS '10), pp. 2169-2173, March 2010.

[13] K. K. Wong and T. K. Ho, "Dynamic coast control of train movement with genetic algorithm," International Journal of Systems Science, vol. 35, no. 13-14, pp. 835-846, 2004.

[14] P. G. Howlett, P. J. Pudney, and X. Vu, "Local energy minimization in optimal train control," Automatica, vol. 45, no. 11, pp. 2692-2698, 2009.

[15] R. Liu and I. M. Golovitcher, "Energy-efficient operation of rail vehicles," Transportation Research A, vol. 37, no. 10, pp. 917-932, 2003.

[16] E. Khmelnitsky, "On an optimal control problem of train operation," IEEE Transactions on Automatic Control, vol. 45, no. 7, pp. 1257-1266, 2000.

[17] Y. Zhang, S. F. Jin, and W. Y. Yue, "An enhanced energy saving strategy for an active DRX in LTE wireless networks," International Journal of Innovative Computing, Information and Control, vol. 9, no. 11, pp. 4373-4387, 2013.

[18] S. Ali and D. Kim, "Energy conservation and comfort management in building environment," International Journal of Innovative Computing, Information and Control, vol. 9, no. 6, pp. 2229-2244, 2013.

[19] Z. Wu, M. Cui, P. Shi, and H. Karimi, "Stability of stochastic nonlinear systems with controlled state-dependent switching," IEEE Transactions on Automatic Control, vol. 58, no. 8, pp. 19041918, 2013.

[20] R. Wang, P. Shi, Z. Wu, and Y. Sun, "Stabilization of switched delay systems with polytopic uncertainties under asynchronous switching," Journal of the Franklin Institute, vol. 350, no. 8, pp. 2028-2043, 2013.

[21] R. Wang, Z.-G. Wu, and P. Shi, "Dynamic output feedback control for a class of switched delay systems under asynchronous switching," Information Sciences, vol. 225, pp. 72-80, 2013.

[22] X. Xu and P. J. Antsaklis, "Optimal control of switched systems based on parameterization of the switching instants," IEEE Transactions on Automatic Control, vol. 49, no. 1, pp. 2-16, 2004.
[23] S. C. Bengea and R. A. DeCarlo, "Optimal control of switching systems," Automatica, vol. 41, no. 1, pp. 11-27, 2005.

[24] Z. Y. Yu, "Train operation experimental report in Dalian fast track," Tech. Rep., Beijing Jiaotong University. 


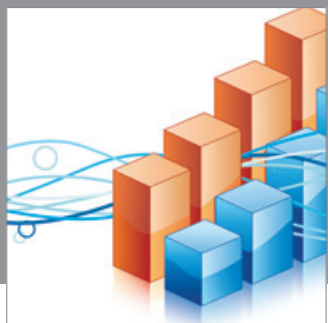

Advances in

Operations Research

mansans

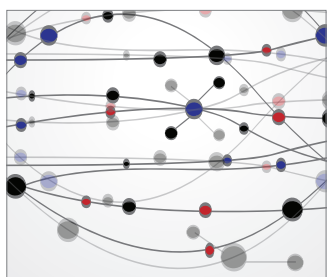

The Scientific World Journal
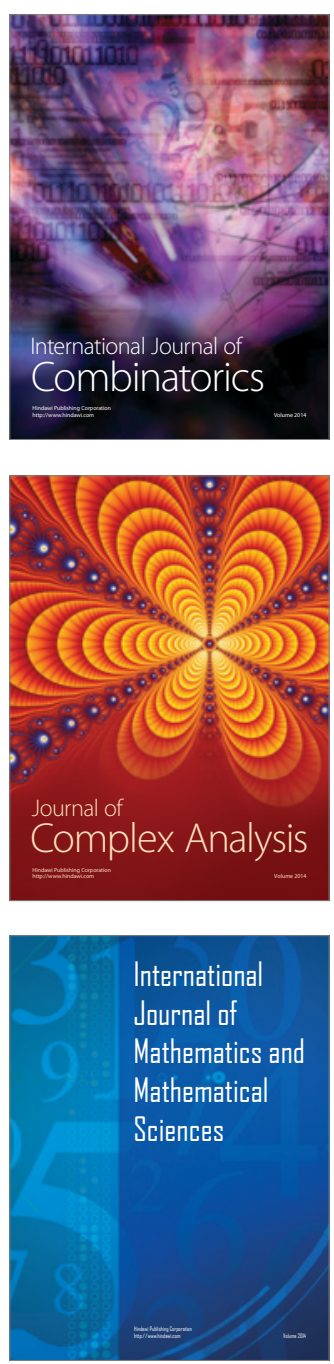
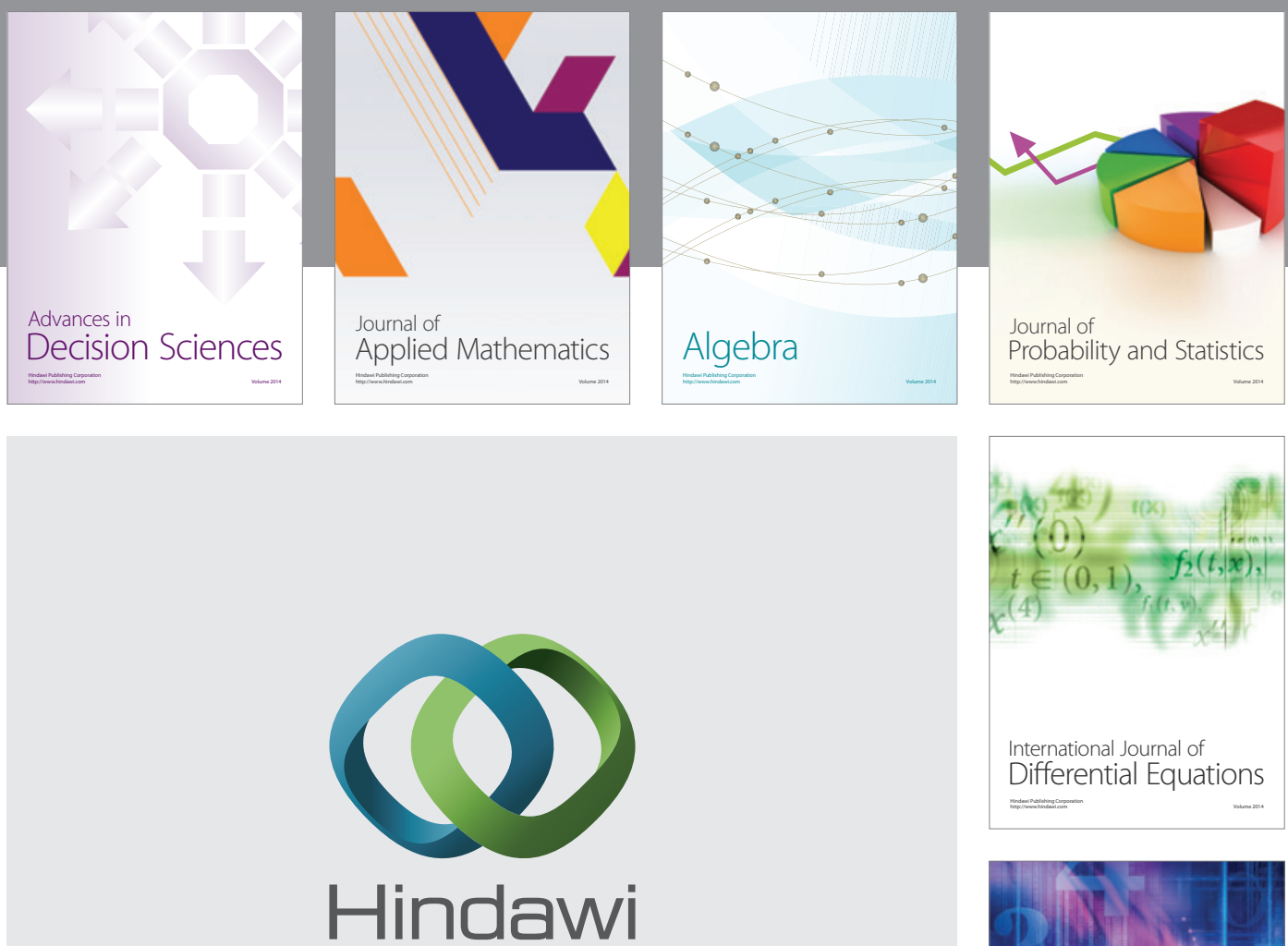

Submit your manuscripts at http://www.hindawi.com
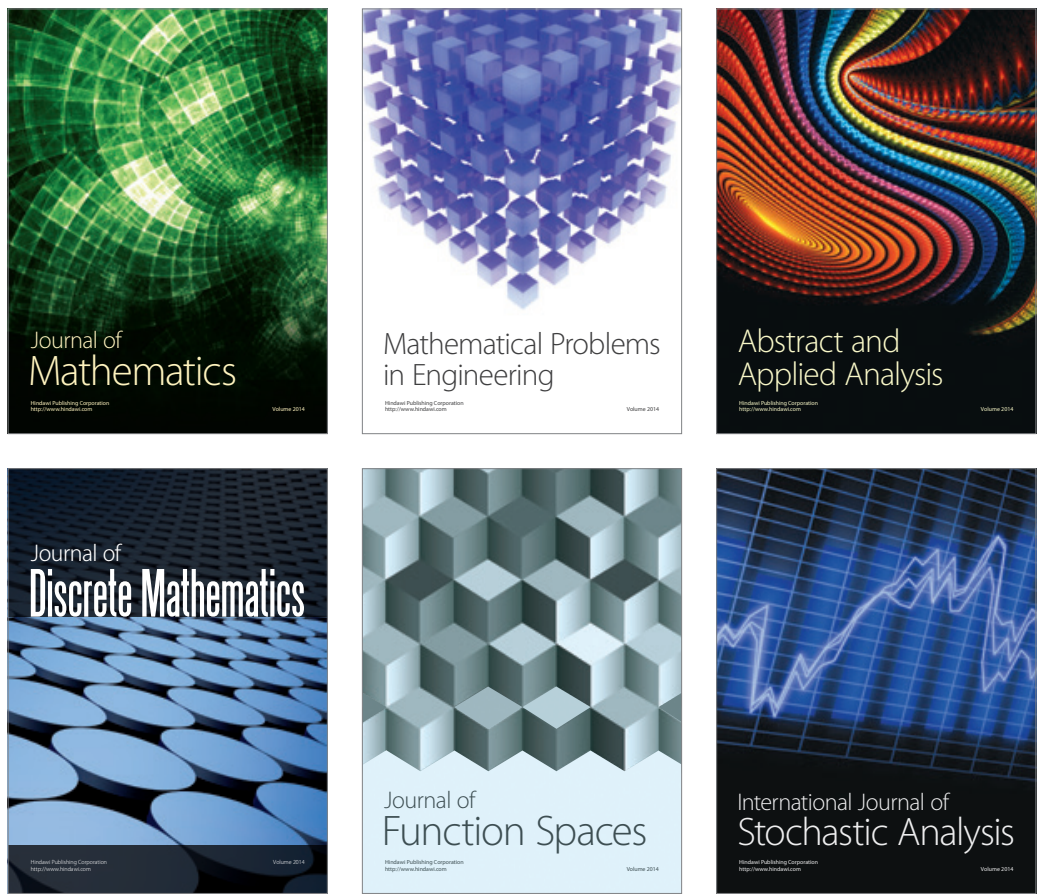

Journal of

Function Spaces

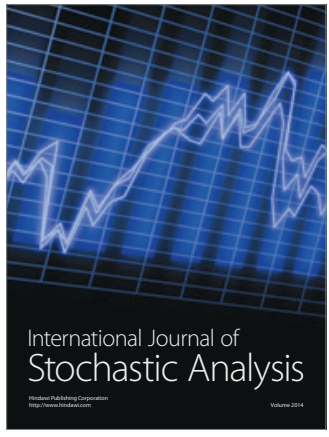

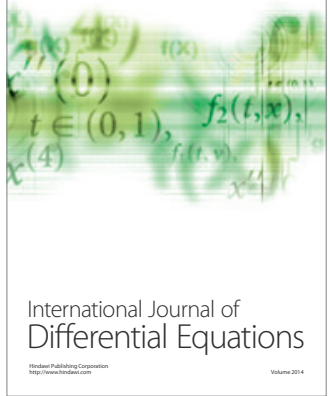
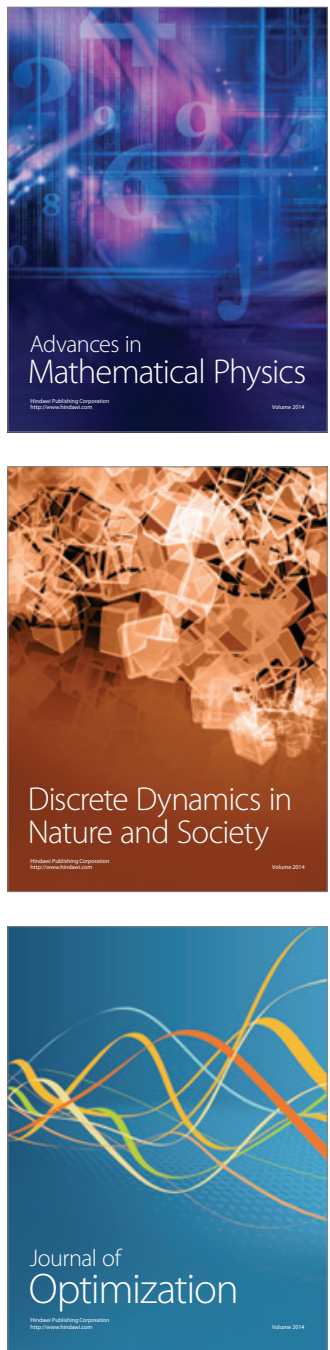